\title{
MENDELIAN INHERITANCE
}

\author{
Helen M Kingston
}

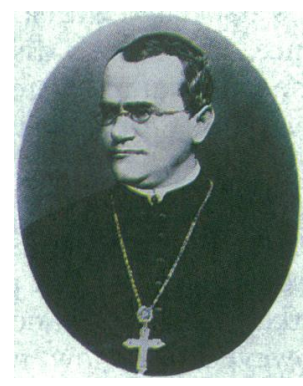

Gregor Mendel 1822-84

\section{Autosomal dominant disorders}
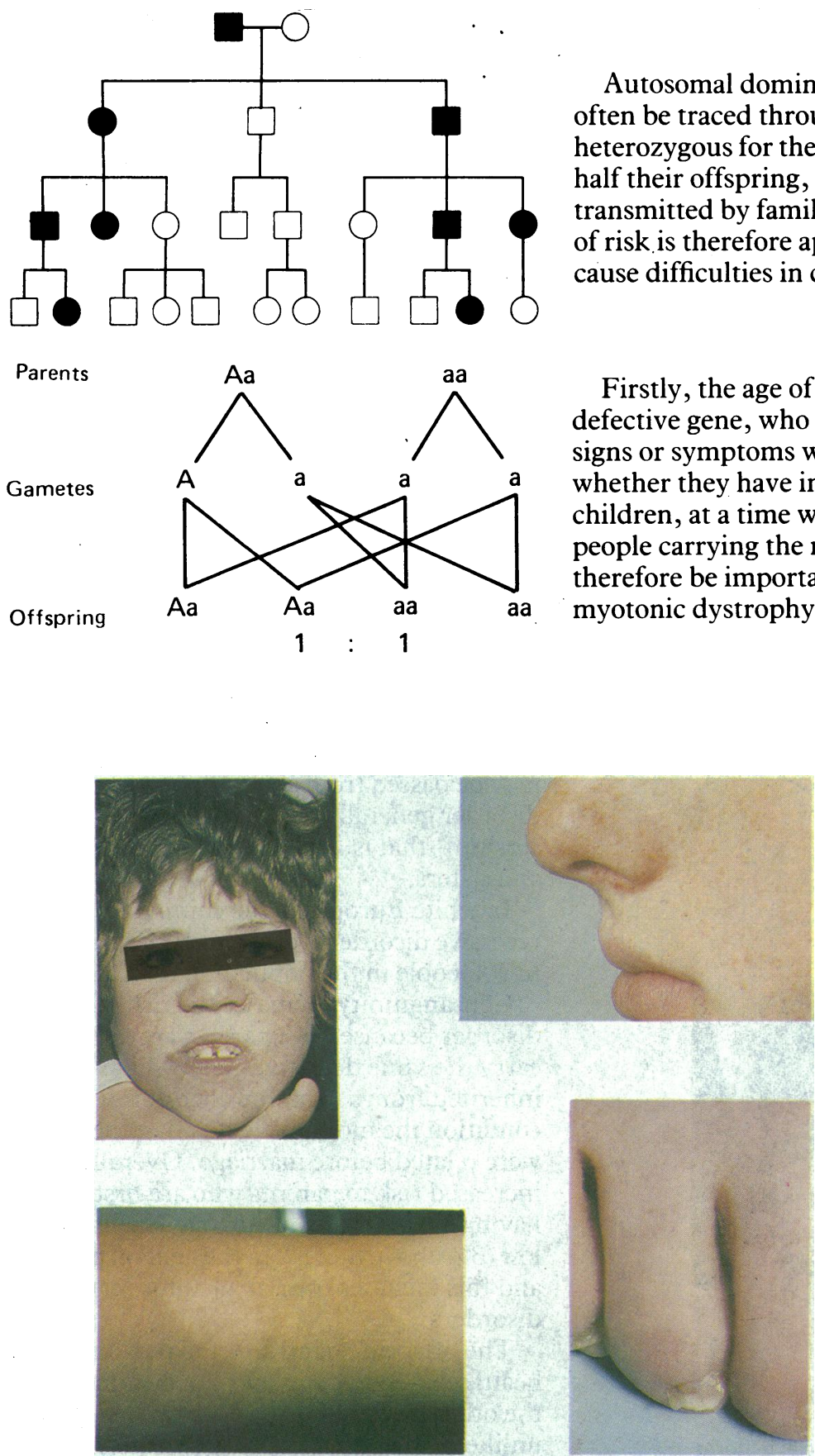

Autosomal dominant disorders affect both males and females and can often be traced through many generations of a family. Affected people are heterozygous for the abnormal allele and transmit the gene for the disease to half their offspring, whether male or female. The disorder is not transmitted by family members who are unaffected themselves. Estimation of risk is therefore apparently simple, but in practice several factors may cause difficulties in counselling families.

Firstly, the age of onset of a disorder may be variable and people with a defective gene, who are destined to become affected, may remain without signs or symptoms well into adult life. Young people at risk may not know whether they have inherited the disorder and will transmit it to their children, at a time when they are planning their own families. Detection of people carrying the mutant gene before symptoms become apparent may therefore be important in conditions such as Huntington's chorea and myotonic dystrophy.

Tuberous sclerosis. Top left: severely affected boy with fits and mental retardation; top right: adenoma sebaceum; bottom left: ash leaf depigmentation; bottom right: periungal fibroma 


\section{Examples of autosomal dominant disorders}

Achondroplasia

Acute intermittent porphyria

Adult polycystic kidney disease

Alzheimer's disease (some cases)

Epidermolysis bullosa (some forms)

Facioscapulohumeral dystrophy

Familial hypercholesterolaemia
Huntington's chorea

Myotonic dystrophy

Noonan's syndrome

Neurofibromatosis

Osteogenesis imperfecta (some forms)

Polyposis coli

Tuberous sclerosis taken to exclude a mild form of the condition in one or other parent before giving this reassurance. New mutation accounts for most cases of achondroplasia, a condition that can be easily excluded in the parents. On the other hand, neurofibromatosis may arise by new mutation or be present in mild form in one parent. In dominant conditions an apparently normal parent may occasionally carry a germline mutation; this is associated with a considerable risk of recurrence. A dominant disorder in a person with a negative family history may alternatively indicate non-paternity.

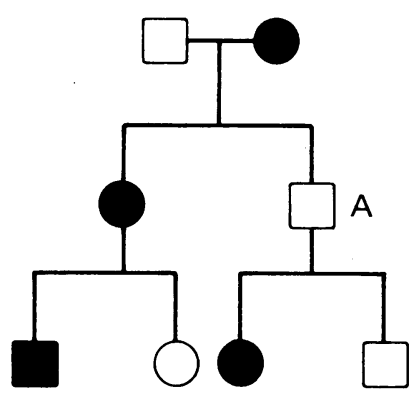

Lack of penetrance in $A$

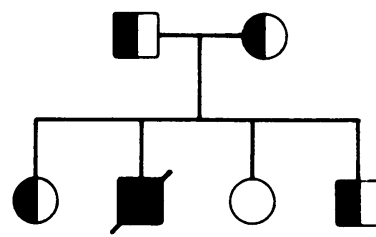

Homozygosity for a dominant disorder

\section{Autosomal recessive disorders}
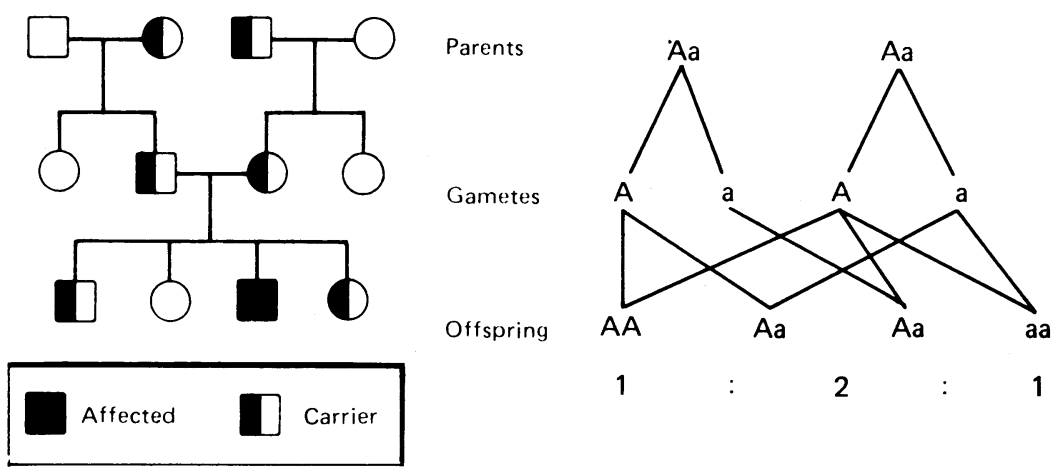

1

2

1
Homozygous affected

Heterozygous affected

A few dominant disorders show lack of penetrance - that is, a person who inherits the gene does not develop the disorder. In this case people who are not affected cannot be completely reassured that they will not transmit the disorder to their children. The risk is, however, fairly low, not exceeding $10 \%$, because when penetrance is high an unaffected person is unlikely to be a gene carrier, and when it is low the chance of a gene carrier developing the disorder is correspondingly small.

Non-genetic factors may also influence the expression of dominant genes - for example, diet in hypercholesterolaemia and drugs in porphyria.

Homozygosity for dominant genes is uncommon, unless two people with the same disorder marry. This may happen preferentially with certain conditions, such as achondroplasia. Homozygous achondroplasia is a lethal condition and the risks for offspring are therefore: $25 \%$ homozygous affected (lethal); $50 \%$ heterozygous affected; $25 \%$ homozygous normal. Thus two out of three living children will be affected.
Autosomal recessive disorders occur in a person whose healthy parents both carry the same recessive gene. The risk of recurrence for future offspring of such parents is $25 \%$. Unlike autosomal dominant disorders there is generally no family history. Although the defective gene may be passed from generation to generation, the disorder generally only appears within a single sibship - that is, within one group of brothers and sisters.

In white Europeans the commonest autosomal recessive disorder is cystic fibrosis, and about one in 20 people in the population is a carrier.

Consanguinity increases the risk of a recessive disorder because both parents are more likely to carry the same defective gene, which has been inherited from a common ancestor. The rarer the condition the more likely it is that the parents were related before marriage. Overall, the increased risk to parents who are first cousins of having a child with severe abnormalities is fairly low ( $3 \%$ above the risk in the general population), and this includes the risk of autosomal recessive disorders.

The offspring of an affected person will be healthy heterozygotes and can be affected only if the other parent is also a gene carrier. This is unlikely except in a consanguineous marriage.
Consanguinity and autosomal recessive inheritance
Hurler's syndrome: coarsening of facial features 


\section{Examples of autosomal recessive disorders}

\section{Congenital adrenal hyperplasia \\ Cystic fibrosis}

Deafness (some forms)

Diastrophic dwarfism

Epidermolysis bullosa

Friedreich's ataxia

Galactosaemia

Haemochromatosis

\section{Homocystinuria}

Hurler's syndrome (mucopolysaccharidosis I)

Laurence-Moon-Biedl syndrome

Occulocutaneous albinism

Phenylketonuria

Sickle cell disease

Tay-Sachs disease

Thalassaemia

\section{$\mathrm{X}$ linked recessive disorders}
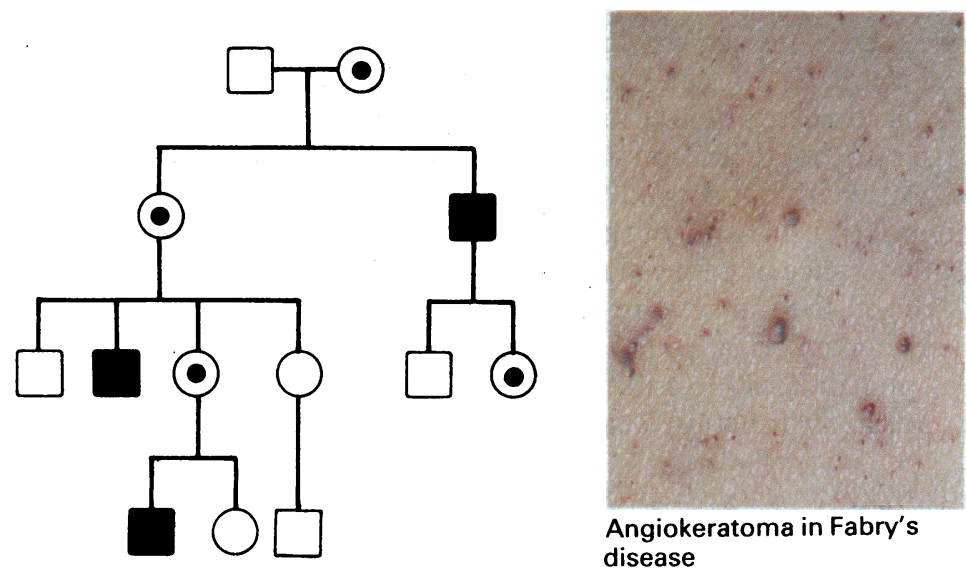

disease

\section{Examples of $X$ linked disorders}

\section{Recessive}

Anhidrotic ectodermal dysplasia

Becker's muscular dystrophy

Colour blindness

Duchenne muscular dystrophy

Fabry's disease
Haemophilia A, B

Hunter's syndrome (mucopolysaccharidosis II)

Menkes's syndrome

Mental retardation with or without fragile site
Lesch-Nyhan syndrome

Glucose-6-phosphate dehydrogenase Occular albinism deficiency

\section{Dominant}

Incontinentia pigmenti

Rickets resistant to vitamin D

Autosomal recessive disorders are commonly severe, and many of the recognised inborn errors of metabolism follow this type of inheritance.

Many complex malformation syndromes are also due to autosomal recessive genes, and their recognition is important in the first affected child in a family because of the $25 \%$ risk of recurrence. Prenatal diagnosis for recessive disorders may be possible by performing biochemical assays or looking for structural abnormalities in the fetus.

In $\mathrm{X}$ linked recessive conditions only males are affected and the disorder is transmitted through healthy female carriers. Occasionally a heterozygous female may show some features of the condition.

A female carrier will transmit the disorder to half her sons, and half her daughters will be carriers. All the daughters of an affected male are obligate carriers whereas none of the sons are affected. X linked recessive disorders cannot be transmitted by a healthy male. Many X linked recessive disorders are severe or lethal during early life, so that the affected males do not reproduce.

An $\mathrm{X}$ linked recessive condition should be considered when the family history indicates affected males in different generations of the family. Family history is, however, not always positive as new mutations are fairly common.

Identifying female gene carriers in the family requires interpretation of the family pedigree and the results of specific tests to identify the carriers.

\section{$\mathrm{X}$ linked dominant disorders}

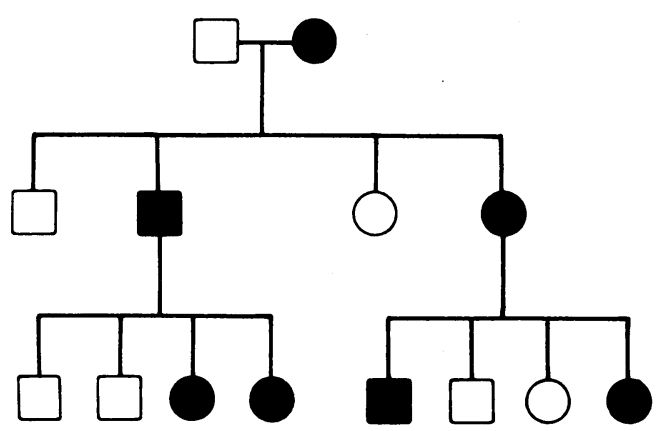

An X linked dominant gene will give rise to a disorder in both hemizygous males and heterozygous females. The gene is transmitted in families in the same way as $\mathrm{X}$ linked recessive genes, giving rise to an excess of affected females. In some disorders the condition is lethal in hemizygous males. In this case there will be fewer males than expected in the family, all of whom will be healthy, and an excess of females, half of whom will be affected. 


\section{Y linked disorders}

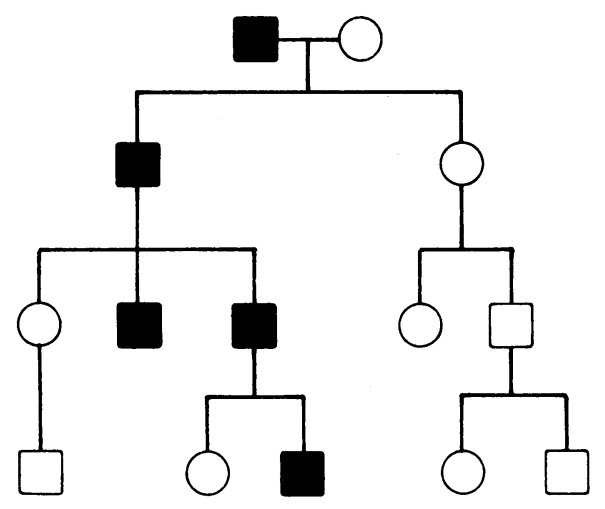

In Y linked disorders only males are affected, with transmission being directly from father to son with the $\mathrm{Y}$ chromosome. This pattern of inheritance has been suggested for such conditions as porcupine skin, hairy ears, and webbed toes. In most conditions in which $Y$ linked inheritance has been postulated the actual mode of inheritance is probably autosomal dominant, with other factors causing sex limitation.

\section{Cytoplasmic inheritance}

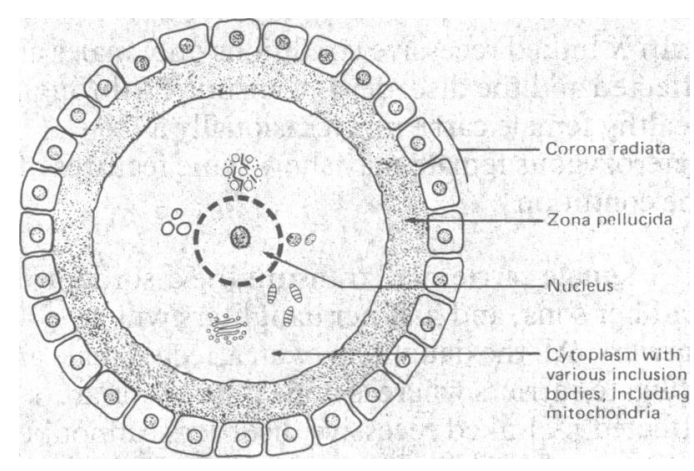

Diagrammatic representation of human egg
Unlike sperm, the egg contains cytoplasm as well as a nucleus. Certain inherited characteristics are probably influenced or wholly determined by cytoplasmic elements. These may be biochemical factors, mitochondrial DNA, or mitochondria themselves and would always be maternally derived. Although not proved, cytoplasmic inheritance may account for the maternal transmission of the congenital form of myotonic dystrophy, the preponderance of paternal transmission in Huntington's chorea of juvenile onset, the transient myasthenia seen in the offspring of mothers with myasthenia gravis, and the absence of father to son transmission of Leber's optic atrophy. In addition, at least one type of myopathy due to mitochondrial dysfunction is maternally transmitted.

\section{McKusick VA. Mendelian inheritance in man. Catalogs of autosomal dominant, autosomal recessive and $X$-linked phenotypes. 8th ed. Baltimore: John Hopkins, 1988.}

The illustrations of tuberous sclerosis were reproduced by kind permission of Professor P S Harper, Institute of Medical Genetics for Wales, Cardiff.

Dr Helen M Kingston, MD, is consultant clinical geneticist at St Mary's Hospital, Manchester.

\section{ANY QUESTIONS}

In general practice antibiotics are usually prescribed in sufficient quantity for five days' treatment and dispensed with an injunction to take the whole course. Is there a sound scientific basis to this advice?

The "appropriate" length of antimicrobial treatment may vary depending on diagnosis, complicating factors, the age of the patient, and the clinical setting. For some infections lengthy treatment is appropriate, so that osteomyelitis or endocarditis may require many weeks' chemotherapy. In uncomplicated urinary tract infection a single dose may suffice. ${ }^{12}$ It is desirable to minimise the length of treatment so far as is consistent with attaining the objective. Objectives may vary: remission of symptoms, defervescence, and return of some laboratory or radiological feature to normal are examples.

In hospital it may be easy to arrange for chemotherapy to be stopped in a day or two if clinical objectives have been achieved. Since this may not be readily arranged in general practice a pragmatic choice of the length of treatment based on clinical experience is often made, five to seven days often being selected for urinary or respiratory infections. While this is often effective, it also commonly represents overtreatment, which is regrettable because it inconveniences the patient or the relatives, may give rise to adverse events, adds to selective pressures for antibiotic resistance in bacteria, and increases costs.

The common injunction to finish the course, often not complied with by patients because they feel better and forget, is probably intended to minimise the emergence of resistance. This supposed motivation is ill founded because the index pathogen is unlikely to become resistant at the site of infection because of underdosing. The emergence of resistant strains of bacteria usually occurs in the commensal flora, and its extent is related to the amount of drug ingested. Short courses are to be preferred. If the injunction to complete the course is not given for that reason it is merely an exercise in unscientific authoritarianism. $-\mathrm{R}$ N GRÜNEBERG, consultant microbiologist, London

1 Harbord RB, Grüneberg RN. Treatment of urinary tract infection with a single dose of amoxycillin, co-trimoxazole, or trimethoprim. Br Med f 1981;283:1301-2.

Bailey RR. Single dose therapy of urinary tract infection. Sydney: ADIS Health Science Press, 1983:125.

If a patient taking $\beta$ blockers develops anaphylactoid shock would adrenaline be recommended despite their interaction or, if not, is there an alternative?

The beneficial effects of adrenaline in anaphylaxis depend on both $\alpha$ and $\beta$ receptors. Stimulation of $\alpha$ receptors induces an increase in blood pressure in the shocked patient by promoting arteriolar constriction. It probably also reduces capillary permeability. Cells that release humoral factors important in the genesis of anaphylaxis are stabilised by a $\beta_{2}$ adrenergic action, and bronchodilatation relies on the same mechanism. These $\beta$ mediated effects must be attenuated when treatment is given to a patient taking $\beta$ blockers, especially if the agent is not a so called selective one. I do not know, however, of any clinical evidence that $\beta$ blockade increases the dangers of anaphylaxis or reduces the efficacy of treatment. Adrenaline remains the treatment of choice, but a $\beta_{2}$ agonist such as salbutamol could be given as an additional agent in severe cases. In appropriate doses this should help to overcome any $\beta_{2}$ blockade that may be hindering recovery.-D A CHAMBERLAIN, consultant cardiologist, Brighton

Skidmore IF. Beta-agonists as mast cell stabilizers. In: Kay AB, ed. Asthma: clinical pharmacology and therapeutic progress. Oxford: Blackwell, 1986. 